\title{
A mathematical model of the apparent mass of the human body under fore-and-aft whole-body vibration
}

\author{
N. Nawayseh \\ Mechanical Engineering Department, College of Engineering, University of Sharjah, \\ P.O. Box 27272 Sharjah, United Arab Emirates \\ Email: nnawayseh@yahoo.co.uk \\ Phone: +97165053947; Fax: +97165585191
}

\begin{abstract}
Studies of biodynamic responses to vibration have mainly focused on vertical vibration, despite the presence of high fore-and-aft and lateral vibration in some vibration environments. Consequently, models of the biodynamic responses to vibration in directions other than vertical are rare. This paper presents a model of the apparent mass of the human body during fore-and-aft vibration. The model is a two-degrees-of-freedom lumped parameter linear model with translational and rotational capabilities. The parameters of the model were optimised using experimentally measured apparent mass. The model gave a close fit to the measured apparent masses of 12 subjects. The parameters of the rotational degrees-of-freedom showed high variability, reflecting the high variability found in the measured data for the 12 subjects. Sensitivity analysis showed that the first peak of the apparent mass is produced by rotational motion while the second peak is caused by translational motion. This study showed that the fore-andaft apparent mass of the human body can be represented by a two-degrees-of-freedom system with rotational and translational capabilities. The proposed model can be integrated with a mathematical model of a seat to predict the transmissibility of the seat. Alternatively, the model parameters can be used to build a mechanical dummy that can be used experimentally for seat testing applications instead of using a human subject.
\end{abstract}

Keywords: Whole-body fore-and-aft vibration; apparent mass; mathematical lumped parameter model.

\section{INTRODUCTION}

Vibration can occur as a translational motion along the fore-and-aft lateral, and/or vertical directions, and/or as a rotational motion about the fore-and-aft axis (roll motion), lateral axis (pitch motion), and/or vertical axis (yaw motion). In real life, whole-body vibration is more likely to occur in more than one direction simultaneously than in a single direction. Depending on the application, the vibration in one direction (or more) could be dominant and more significant than in the other directions. For example, trains have high horizontal motion (e.g. Nuawi et al. [1], Gong and Griffin [2] ), ships have substantial rotational motion (e.g. Griffin [3]) and off-road vehicles have high horizontal vibration components, as reported by Lundström and Lindberg [4]. Some studies have also shown that the perception thresholds during horizontal excitation in the range 1 to $10 \mathrm{~Hz}$ are similar to those during vertical excitation (subjective studies summarised by Griffin [3]). Despite that, the vast majority of the studies of single axis vibration have focused on vibration in the vertical direction (e.g. [5-10]), while only a few studies have investigated 
the biodynamic responses to vibration in directions other than the vertical direction (e.g. [8-11]).

Owing to the abundance of data for biodynamic responses to vertical vibration, many studies have attempted to model the biodynamic responses to whole-body vertical vibration (e.g. $[12,13])$. Very few models of biodynamic responses to vibrations in other directions are available in the literature. The fore-and-aft models reported in the literature are either with translational capability only $[14,15]$ or with translational and rotational capabilities but representing the apparent mass at the back only. Mansfield and Lundström [14] presented six different lumped parameter models ranging from a single to three degrees of freedom with only translational motion capability. The models were used to separately represent the fore-and-aft and lateral apparent masses. The parameters of the models were obtained by calibrating the responses of the models using experimentally measured median apparent masses. The best agreement between the response of the model and the measured data was obtained when using a three-degrees-of-freedom model that consisted of three parallel single-degree-of-freedom systems attached to a rigid support. However, the phase response of the model was in agreement with the measured phase angle up to $4 \mathrm{~Hz}$ only. Stein, Múčka [15] developed an integrated person-cushioned seat lumped parameter model with translational capability only. The fore-and-aft apparent mass was used to optimise all parameters of the integrated person-seat model. The model was used to study the reaction at the steering wheel in the driver position and it was concluded that the steering wheel reaction has to be considered for a driver sitting in a cushioned suspended driver's seat. Qiu and Griffin [16] developed a lumped parameter model with translational and rotational degrees of freedom to represent the fore-and-aft apparent mass of the back. The model was integrated with a car seat model in order to predict the transmissibility of the backrest under fore-and-aft vibration. The human body model was calibrated using the fore-and-aft apparent mass of the back measured previously by Nawayseh and Griffin [11]. Qiu and Griffin [16] concluded that the model was able to represent the measured apparent masses at the back and predict the transmissibility of the backrest of the car seat.

The literature review showed evidence of high vibration in the horizontal direction (fore-and-aft and lateral) in some vibration environments. Nevertheless, there are only a few experimental and modelling studies in the literature that report the biodynamic response (e.g. driving point mechanical impedance, apparent mass or transmissibility) of the human body to horizontal vibration. The objective of this work is to develop a mathematical model that can represent the apparent mass of the human body under foreand-aft vibration. Since previous experimental studies generally showed two peaks in the fore-and-aft apparent mass [10], it was hypothesised that a two-degrees-of-freedom model will be adequate to represent the fore-and-aft apparent mass. A sensitivity analysis will be performed so as to identify the part(s) of the model that are responsible for the different peaks appearing in the experimentally measured apparent mass. In addition to quantitatively summarising experimental data in a mathematical model, such a model will be useful for testing the performance and predicting the transmissibility of new vehicle seats, as will be explained later in this paper.

\section{METHOD}

\section{Experimental measurements}

The fore-and-aft apparent mass measured by Nawayseh and Griffin [10] was used to calibrate the model. In their experiments, Nawayseh and Griffin [10] exposed 12 male 
seated subjects to whole-body random fore-and-aft vibration with a magnitude of 1.25 $\mathrm{ms}^{-2}$ r.m.s. Figure 1 shows a schematic diagram of the experimental setup. The experiment was conducted with the subjects sitting on a flat rigid seat with no back support while adopting two positions one at a time, namely feet-supported posture and feet-unsupported posture. In the feet-supported posture, the subjects placed their feet on an adjustable footrest (which moved in phase with the seat) such that the upper legs were horizontal while the lower legs were vertical (Figure 1). In the feet-unsupported posture, the footrest shown in Figure 1 was removed and the feet were not supported. With both positions, the subjects were instructed to sit in an upright (but not erect) upper body posture and place their hands in their laps.

The fore-and-aft force on the seat was measured using a Kistler 9281 B force platform (see Figure 1) and the fore-and-aft acceleration was measured using a piezoresistive accelerometer type Entran EGCSY-240D-10 as reported by Nawayseh and Griffin [10]. The fore-and-aft force and the fore-and-aft acceleration were acquired via an HVLab signal processing and data acquisition system and then saved on a computer for analysis. The fore-and-aft apparent mass was then calculated from the complex ratio of force to acceleration. More details of the experimental setup can be found in the work by Nawayseh and Griffin [10].

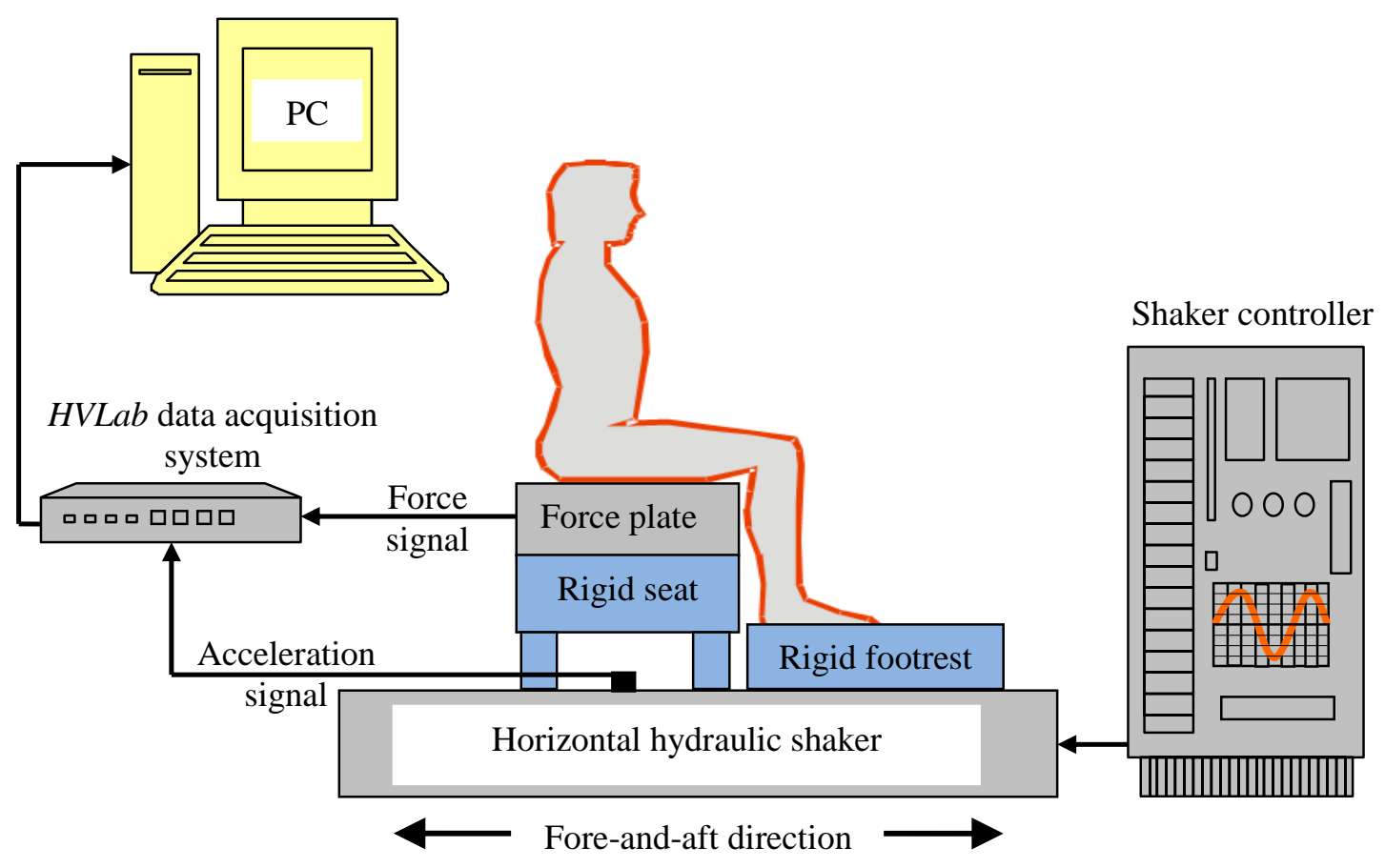

Figure 1 Experimental setup used by Nawayseh and Griffin [10] to measure the apparent mass. The setup is shown for the feet-supported position.

\section{Model description}

The experimentally measured fore-and-aft apparent mass showed two peaks for most subjects, as presented by Nawayseh and Griffin [10], which implies the need for a twodegrees-of-freedom model to reproduce the experimental data. Hence, a two-degrees-offreedom lumped parameter model is proposed in this study. The model is obviously not intended to represent the full complexity of motions occurring in the seated subjects during fore-and-aft excitation but rather the gross response of the human body on the seat. 
Nevertheless, to be close to the type of body motions experienced by seated subjects under fore-and-aft vibration, it was decided to build a model with rotational and translational motion capabilities (Figure 2) instead of a relatively simpler system of translational motion. The model consists of two masses $\left(m_{1}\right.$ and $\left.m_{2}\right)$, a translation spring and damper with stiffness and damping coefficients of $k_{\mathrm{x}}$ and $c_{\mathrm{x}}$, respectively, and a rotational spring and damper with stiffness and damping coefficients of $k_{\mathrm{r}}$ and $c_{\mathrm{r}}$, respectively. As shown in Figure 2, when fore-and-aft vibration is introduced at the base of the model, mass 1 undergoes translational (fore-and-aft) motion causing mass 2 to undergo both translational (fore-and-aft) motion and rotational (pitch) motion. Previous experimental studies showed evidence of high forces on the seat in the vertical direction under pure fore-and-aft vibration, indicating a pitch motion of the body or some parts of the body [10]. The proposed model structure (or more likely a modified version of it) can be used in future work to reproduce the vertical forces (i.e. cross-axis apparent mass) measured on the seat during fore-and-aft vibration.
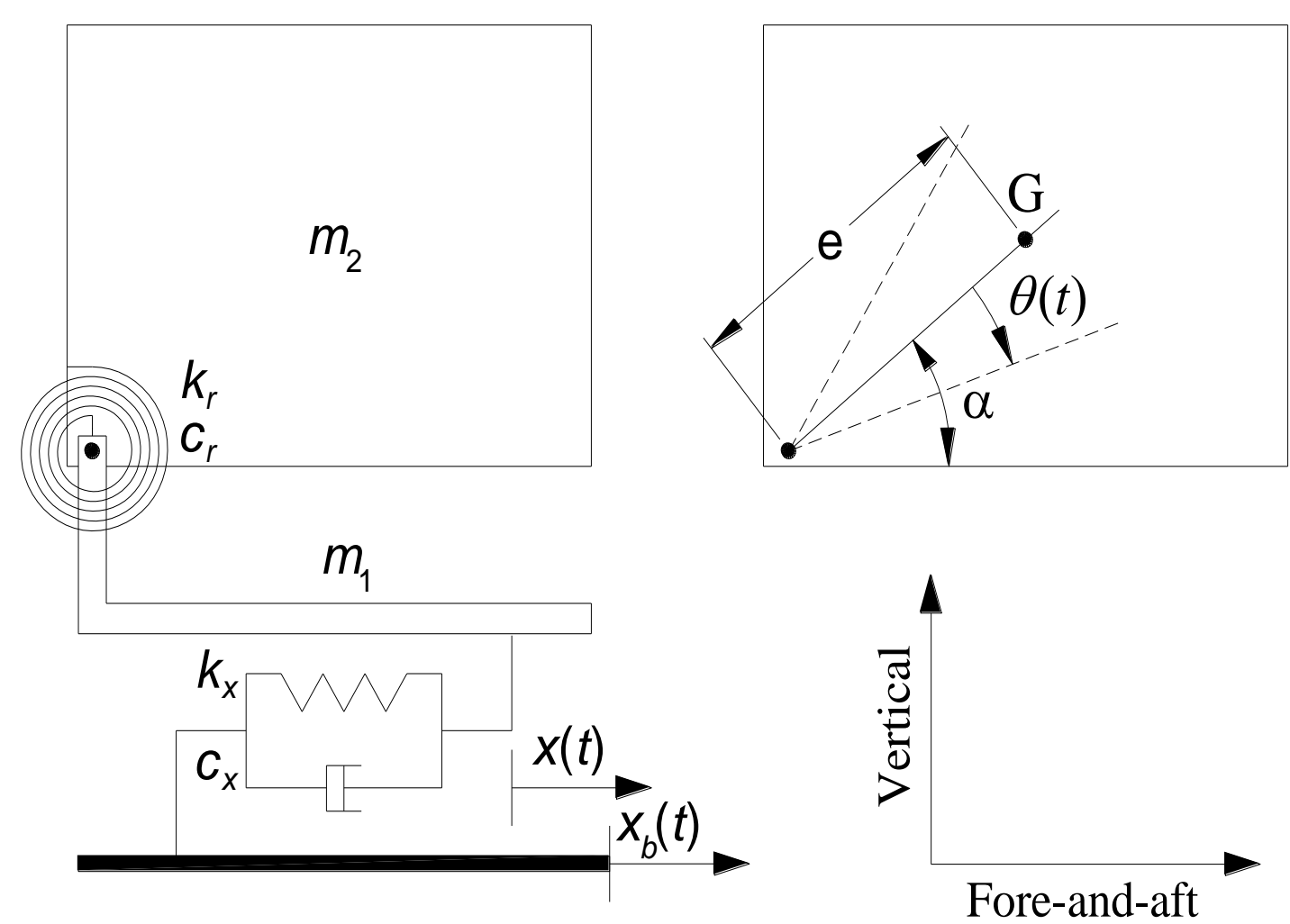

Figure 2 The proposed two-degrees-of-freedom model with translational and rotational

\section{Equations of motion} capabilities.

The equations of motion of the model are given by Eq. (1) and Eq. (2) as follows:

$$
\begin{gathered}
m_{1} \frac{\mathrm{d}^{2} x}{\mathrm{~d} t^{2}}+m_{2}\left(\frac{\mathrm{d}^{2} x}{\mathrm{~d} t^{2}}+e \sin \alpha \frac{\mathrm{d}^{2} \theta}{\mathrm{d} t^{2}}\right)+c_{\mathrm{x}}\left(\frac{\mathrm{d} x}{\mathrm{~d} t}-\frac{\mathrm{d} x_{\mathrm{b}}}{\mathrm{d} t}\right)+k_{\mathrm{x}}\left(x-x_{b}\right)=0 \\
\left(m_{2} e^{2}+J_{2}\right) \frac{\mathrm{d}^{2} \theta}{\mathrm{d} t^{2}}+m_{2} e \sin \alpha \frac{\mathrm{d}^{2} x}{\mathrm{~d} t^{2}}+c_{\mathrm{r}} \frac{\mathrm{d} \theta}{\mathrm{d} t}+k_{\mathrm{r}} \theta=0
\end{gathered}
$$


The total fore-and-aft force $\left(f_{x}(t)\right)$ produced in the model can be found from Eq. (3):

$$
f_{x}(t)=m_{1} \frac{\mathrm{d}^{2} x}{\mathrm{~d} t^{2}}+m_{2}\left(\frac{\mathrm{d}^{2} x}{\mathrm{~d} t^{2}}+e \sin \alpha \frac{\mathrm{d}^{2} \theta}{\mathrm{d} t^{2}}\right)
$$

where:

$m_{1}$ and $m_{2}$ are the masses of mass 1 and mass 2, respectively.

$k_{\mathrm{x}}$ and $c_{\mathrm{x}}$ are the fore-and-aft stiffness and damping coefficients beneath mass 1 , respectively.

$k_{\mathrm{r}}$ and $c_{\mathrm{r}}$ are the rotational stiffness and damping coefficients of mass 2 , respectively.

$J_{2}$ is the moment of inertia of mass 2 about its centre of mass.

$e$ is the distance between the centre of mass of mass 2 and the revolute joint.

$\alpha$ is the angle that $e$ has with the horizontal when the model is in equilibrium.

$x$ represents the fore-and-aft degrees-of-freedom

$\theta$ represents the rotational degrees-of-freedom (rotational motion of mass 2 ).

$x_{b}$ represents the horizontal (fore-and-aft) motion of the seat where the force and acceleration used to calculate the apparent mass were measured.

The apparent mass can be calculated by taking the Laplace transform of the above equations assuming zero initial conditions and solving it for the complex ratio between $\mathrm{F}_{x}(s)$ and $\mathrm{s}^{2} \mathrm{X}_{b}(s)$, where $\mathrm{F}_{x}(s)$ is the Laplace transform of the total force, $f_{x}(t), \mathrm{X}_{b}(s)$ is the Laplace transform of the displacement, $x_{b}(t)$, and $s$ is $j \omega$ where $j$ is $(-1)^{1 / 2}$ and $\omega$ is the angular frequency. The apparent mass $(A M)$ can then be calculated using Eq. (4):

$$
A M=\left(m_{1}+m_{2}\right) \frac{C D}{A D-B^{2}}+m_{2} e \sin \alpha \frac{B C}{B^{2}-A D}
$$

where:

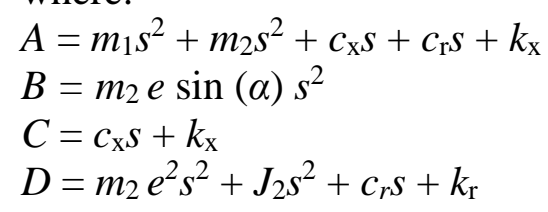

The apparent mass modulus and phase can be calculated from the real and imaginary parts of the apparent mass using Eq. (5) and Eq. (6):

$$
\begin{gathered}
A M_{\text {mod }}(\omega)=\sqrt{(\operatorname{ReAM}(\omega))^{2}+(\operatorname{ImAM}(\omega))^{2}} \\
A M_{\mathrm{ph}}(\omega)=\tan ^{-1} \frac{\operatorname{ImAM}(\omega)}{\operatorname{ReAM}(\omega)}
\end{gathered}
$$

\section{Procedure}

The parameters of the model (i.e. the inertia parameters, stiffness, damping coefficients and the geometrical parameters $e$ and $\alpha$ ) were all optimised using the interior-point algorithm $[17,18]$ within MATLAB 2012b. In order to give them a physical meaning, the optimised parameters of the model were restrained to have positive values only. The parameters of the model were optimised by comparing the experimentally measured moduli and phases of the fore-and-aft apparent mass with the moduli and phases calculated from the model as shown in Eq. (7): 


$$
\text { error }=w_{1} \sum_{i=1}^{N}\left(A M_{\text {mod_c }}-A M_{\text {mod_m }}\right)_{i}{ }^{2}+w_{2} \sum_{i=1}^{N}\left(A M_{\text {ph_c }}-A M_{\text {ph } \_\mathrm{m}}\right)_{i}{ }^{2}
$$

where:

$A M_{\text {mod_c }}$ and $A M_{\text {ph_c }}$ are the modulus and phase of the calculated fore-and-aft apparent masses, respectively.

$A M_{\text {mod_m }}$ and $A M_{\text {ph_m }}$ are the modulus and phase of the measured fore-and-aft apparent masses, respectively.

$w_{1}$ and $w_{2}$ are weighting factors employed to improve the fitting. They were chosen arbitrarily but taking into consideration the difference in the order of magnitude between the modulus and the phase: the weighting factor for the phase was higher than that for the modulus to compensate for the difference in the order of magnitude between the modulus and the phase. The values of those weighting factors varied among the different subjects.

Each of the nine parameters of the model was assigned an initial guess, which was corrected during the optimisation process. The initial guesses for the stiffness and damping coefficients were chosen to be similar to those reported in the literature. In some cases, the initial guesses gave a reasonable curve fitting that was improved further by adjusting the arbitrary weighting factors in the error equation. In other cases, different sets of initial guesses had to be tried so as to get an acceptable match between the experimental data and the model response. In any case, several different sets of initial guesses were tried and the solution was accepted only when the different initial guesses resulted in the same model parameters.

\section{RESULTS AND DISCUSSION}

\section{Fitting of Median Data}

The model was used to represent the median fore-and-aft apparent mass of 12 subjects sitting with two different positions (Figure 3 and Figure 4). The figures show that the model is capable of providing a close fit to both the median apparent mass magnitude and phase for both investigated positions. The parameters of the model obtained through this process are given in the last row of Table 1 for the feet-supported position and in the last row of Table 2 for the feet-unsupported position. The optimisation resulted in zero or a very low value for $m_{1}$ during the fitting of both the median and individual data and hence it was decided to fix the value of $m_{1}$ at zero in the model. Thus, mass 1 can be thought of as a massless frame on which mass 2 is mounted via a revolute joint.

Although the value of $m_{1}$ was set to zero, the value of $m_{2}$ was optimised and was not fixed at the static mass supported on the seat. Unlike the vertical apparent mass, the fore-and-aft apparent mass at low frequencies (below $1 \mathrm{~Hz}$ ) is not equal to the static mass supported on the vibrating surface, indicating a more complex response in the fore-andaft direction than in the vertical direction $[8,10]$. This led some previous researchers to normalise the fore-and-aft apparent mass with respect to the standing mass rather than the static sitting mass [8]. The difference between the fore-and-aft apparent mass at low frequencies and the static sitting mass could be owing to the sway of the upper body during fore-and-aft vibration, which creates a horizontal force component that will affect the fore-and-aft force measured on the seat: when exposed to seat acceleration in the fore direction, the upper body is expected to sway backward and when exposed to aft seat acceleration, the body is expected to sway forward $[10,11]$. Thus, the total force on the seat consists of two forces in opposite directions: one resulting from accelerating the mass and the other resulting from the sway of the upper body. This could be the main reason 
for the difference between the fore-and-aft apparent mass at low frequencies and the static mass supported on the seat. The same reasoning can be used to explain why the optimised value of mass 2 is lower than the apparent mass at low frequency: the equation of the fore-and-aft force of the model consists of a translational motion term and a rotational motion term, and both contribute with different degrees (depending on the frequency) to the calculation of the apparent mass. The sensitivity analysis of the proposed model indicated that the apparent mass at low frequency is influenced by the rotational motion of mass 2, which is consistent with the above explanation. It is also worth mentioning that letting the model find the value of mass 2 gave the optimisation process more flexibility when searching for values for the other parameters.

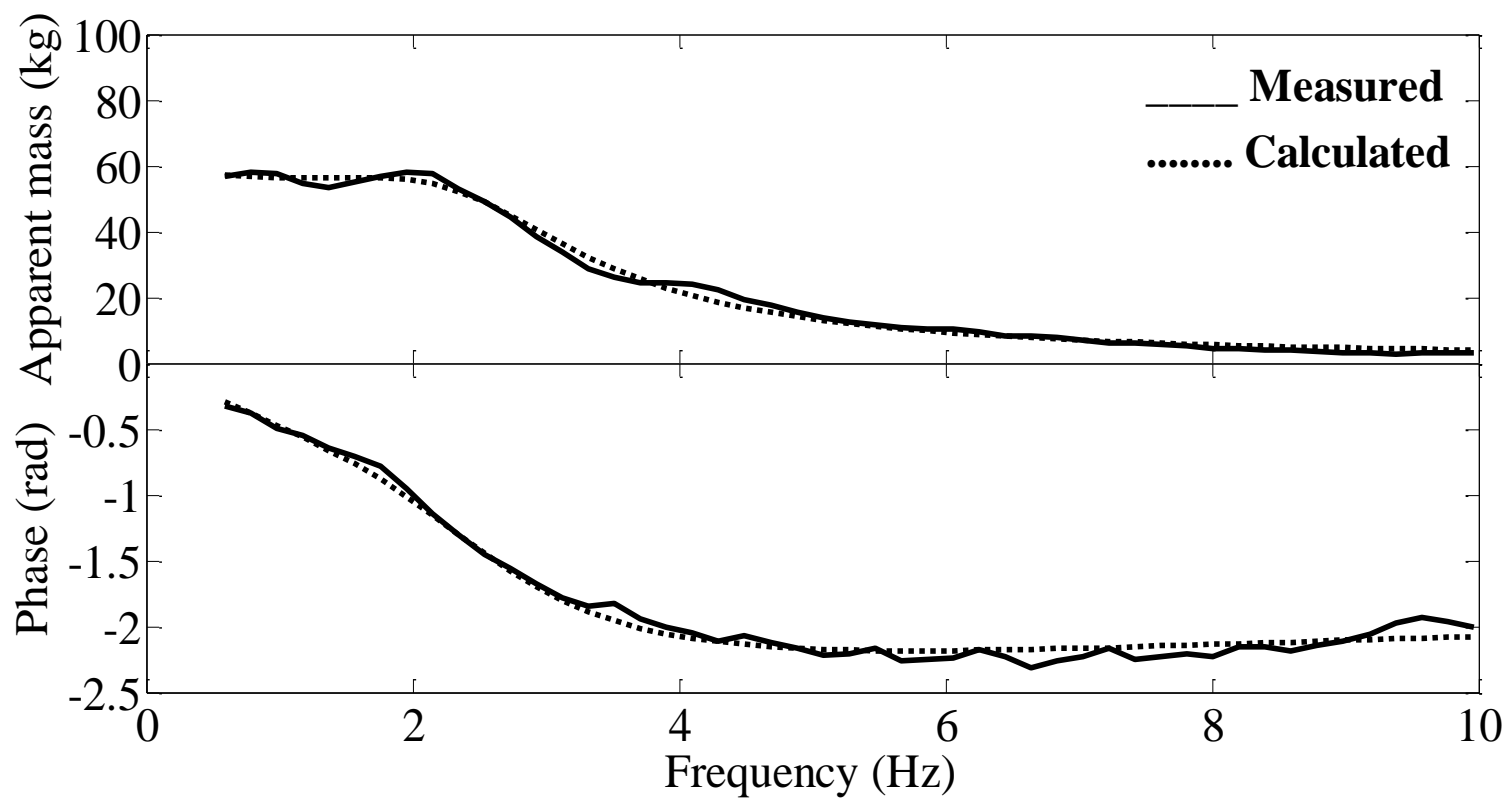

Figure 3. Median fore-and-aft apparent mass for the feet-supported position.

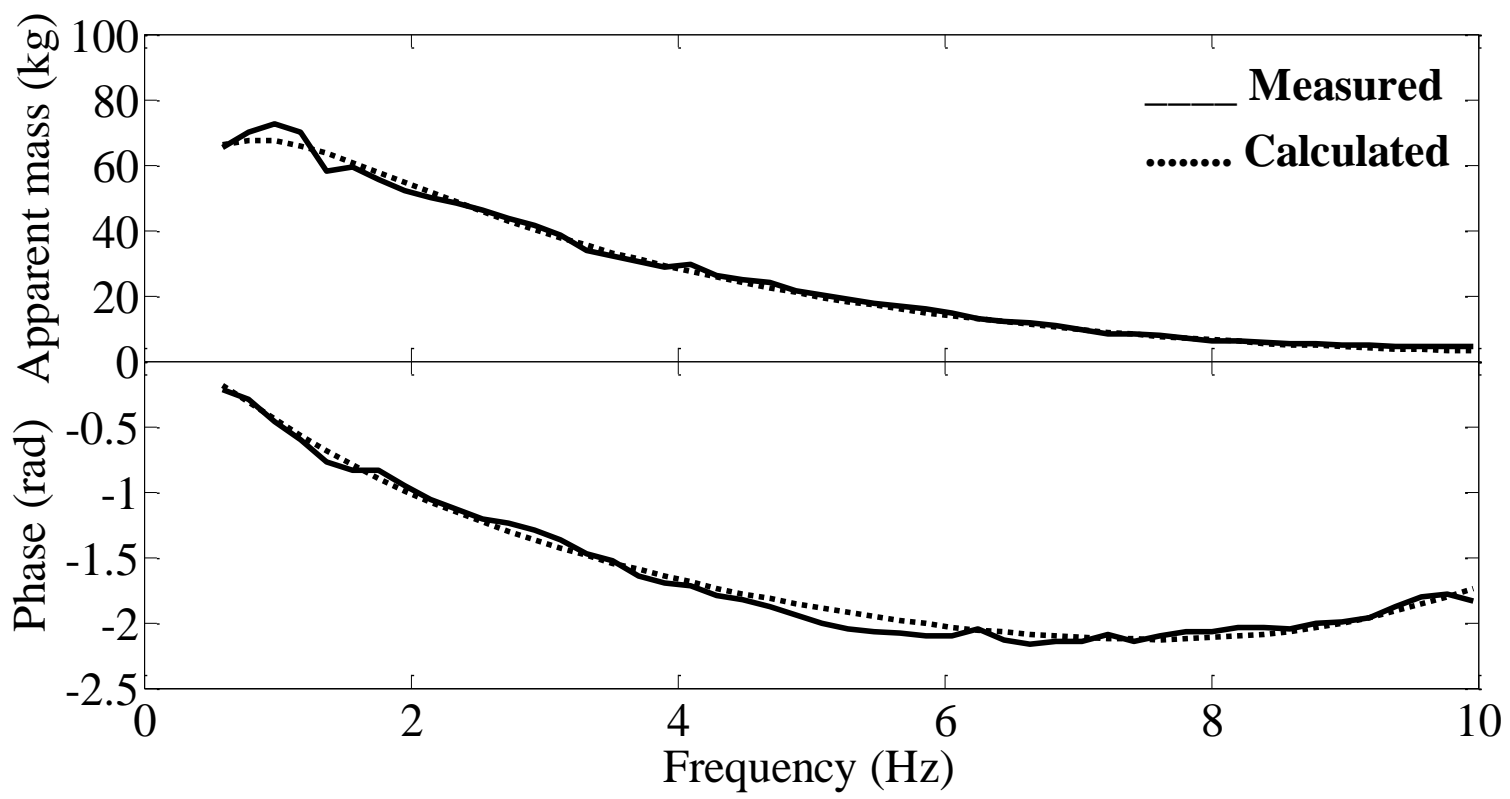

Figure 4. Median fore-and-aft apparent mass for the feet-unsupported position. 
Table 1. Optimised parameters for median and individual responses of 12 subjects for the feet-supported position. $\left(m_{1}=0\right)$.

\begin{tabular}{ccccccccc}
\hline $\begin{array}{c}\text { Sub. } \\
\text { No. }\end{array}$ & $m_{2}$ & $\begin{array}{c}J_{2} \\
\mathrm{~kg}\end{array}$ & $\begin{array}{c}k_{\mathrm{x}} \\
\mathrm{kg} \mathrm{m}\end{array}$ & $\begin{array}{c}k_{\mathrm{r}} \\
\mathrm{N} / \mathrm{m}\end{array}$ & $\begin{array}{c}c_{\mathrm{x}} \\
\mathrm{N} \mathrm{m} / \mathrm{m}\end{array}$ & $\begin{array}{c}c_{\mathrm{r}} \\
\mathrm{N} \mathrm{s} \mathrm{m}\end{array}$ & $\begin{array}{c}e \\
\mathrm{~m}\end{array}$ & $\begin{array}{c}\alpha \\
\mathrm{rad}\end{array}$ \\
\hline 1 & 37 & 5.0 & 7021 & 6 & 139 & 876 & 0.63 & 1.37 \\
2 & 67 & 3.5 & 10164 & 94 & 226 & 64 & 0.30 & 1.33 \\
3 & 44 & 0.8 & 10663 & 4528 & 1114 & 29 & 0.33 & 1.53 \\
4 & 46 & 9.4 & 6908 & 483 & 227 & 68 & 0.33 & 1.13 \\
5 & 55 & 9.7 & 6871 & 1470 & 191 & 221 & 0.74 & 0.85 \\
6 & 76 & 7.2 & 10282 & 21 & 197 & 65 & 0.43 & 1.41 \\
7 & 70 & 0.2 & 9465 & 3 & 213 & 2 & 0.06 & 1.26 \\
8 & 42 & 5.4 & 12251 & 611 & 218 & 127 & 0.54 & 0.85 \\
9 & 75 & 0.1 & 8523 & 1 & 324 & 1 & 0.05 & 1.12 \\
10 & 57 & 0.2 & 2 & 712 & 577 & 8 & 0.25 & 1.55 \\
11 & 43 & 1.8 & 7771 & 5995 & 794 & 64 & 0.72 & 1.55 \\
12 & 72 & 8.8 & 5795 & 182 & 178 & 62 & 0.36 & 1.05 \\
Median & 57 & 5.6 & 8864 & 35.7 & 189 & 142 & 0.49 & 1.10 \\
\hline
\end{tabular}

Table 2. Optimised parameters for median and individual responses of 12 subjects for the feet-unsupported position. $\left(m_{1}=0\right)$.

\begin{tabular}{|c|c|c|c|c|c|c|c|c|}
\hline $\begin{array}{l}\text { Sub. } \\
\text { No. }\end{array}$ & $\begin{array}{l}\boldsymbol{m}_{\mathbf{2}} \\
\mathrm{kg}\end{array}$ & $\begin{array}{c}\boldsymbol{J}_{\mathbf{2}} \\
\mathrm{kg} \mathrm{m}^{2}\end{array}$ & $\begin{array}{c}\boldsymbol{k}_{\mathbf{x}} \\
\mathrm{N} / \mathrm{m}\end{array}$ & $\begin{array}{c}\boldsymbol{k}_{\mathbf{r}} \\
\mathrm{N} \mathrm{m}\end{array}$ & $\begin{array}{c}c_{\mathbf{x}} \\
\mathrm{N} \mathrm{s} / \mathrm{m}\end{array}$ & $\begin{array}{c}\boldsymbol{c}_{\mathbf{r}} \\
\mathrm{N} \mathrm{s} \mathrm{m}\end{array}$ & $\begin{array}{l}\boldsymbol{e} \\
\mathrm{m}\end{array}$ & $\begin{array}{c}\alpha \\
\mathrm{rad}\end{array}$ \\
\hline 1 & 42 & 10 & 7118 & 696 & 239 & 87 & 0.33 & 1.57 \\
\hline 2 & 67 & $3.9 \mathrm{E}-10$ & 27203 & 6 & 217 & 1 & 0.05 & 0.97 \\
\hline 3 & 60 & 0.032 & 3742 & 1707 & 1003 & 0.9 & 0.05 & 1.56 \\
\hline 4 & 45 & 0.0089 & 19278 & 5 & 7.6E-05 & 1 & 0.05 & 1.12 \\
\hline 5 & 66 & $3.7 \mathrm{E}-08$ & 22063 & 7.1E-06 & 59 & $1.2 \mathrm{E}-06$ & 4.4E-05 & 1.37 \\
\hline 6 & 52 & 0.053 & 13265 & 28 & 236 & 5 & 0.09 & 1.03 \\
\hline 7 & 55 & 0.195 & 11992 & 15 & 342 & 2 & 0.05 & 1.16 \\
\hline 8 & 45 & 0.0014 & 11218 & 7 & 239 & 1 & 0.05 & 0.77 \\
\hline 9 & 53 & 0.803 & 13823 & 74 & 274 & 10 & 0.11 & 1.46 \\
\hline 10 & 52 & 0.023 & 10765 & 8 & 196 & 1 & 0.05 & 1.03 \\
\hline 11 & 56 & 5.94 & 8947 & 1186 & 226 & 250 & 0.73 & 0.78 \\
\hline 12 & 66 & 0.885 & 8003 & 48 & 342 & 4 & 0.07 & 1.41 \\
\hline Median & 57 & 1.34 & 2411 & 7352 & 767 & 44 & 0.42 & 1.39 \\
\hline
\end{tabular}

Models that describe the responses of humans to vibration can be categorised into three main categories: mechanistic models, which provide a representation of how the human body moves when exposed to vibration, quantitative models, which summarise biodynamic measurements in a mechanical system, and effect models, which give indications about the effect of motion on human health, comfort and/or performance [19]. For example, effect models could be very useful in studying the relationship between low back pain and vibration exposure along with experimental investigations, such as the study reported by Ismail et al. [20]. The complexity of the model depends on the application for which the model will be used and hence quantitative models are expected 
to be the least complex models among the three categories. The model proposed in this paper is a quantitative mechanical model that represents the fore-and-aft apparent mass of the human body. The usefulness of quantitative models is beyond just being a summary of biodynamic responses. For example, the optimised parameters obtained from fitting the median data in this paper can be useful in evaluating the fore-and-aft transmissibility of compliant seats through integrating the mathematical model of the median data with a mathematical model of the seat (the seat-person system is a coupled system in which the dynamic response of the seat is affected by the biodynamic response of the occupant of the seat and vice versa). This has been done before to study the vertical transmissibility of a car seat during vertical vibration [21] and the fore-and-aft transmissibility of a seat backrest during fore-and-aft vibration [16]. Alternatively, the optimised parameters can be used to build an anthropometric dummy for the evaluation of seat performance under vibration instead of using human subjects. Using an anthropometric dummy has several advantages over using human subjects, such as repeatability, time flexibility and the possibility of performing seat testing experiments under severe vibration conditions. Thus, the proposed model can be a useful reference for vehicle seat manufacturers designing seats with optimum parameters that help reduce the transmission of vibration through the seat.

\section{Fitting of Individual Data}

The model was employed to represent the moduli and phases of the fore-and-aft apparent masses of 12 subjects. With both positions (i.e. the feet-supported and feet-unsupported), the calculated (i.e. from the model) and measured (i.e. from the experiment) apparent masses were in good agreement for both the moduli and phases (Figures 5-8). The experimental data show only one peak in the apparent masses of some subjects and two peaks in the apparent masses of other subjects. The model replicates the two peaks in the data. Some subjects showed a third peak with low magnitude; however, being a twodegrees-of-freedom model, the model can produce only two peaks.

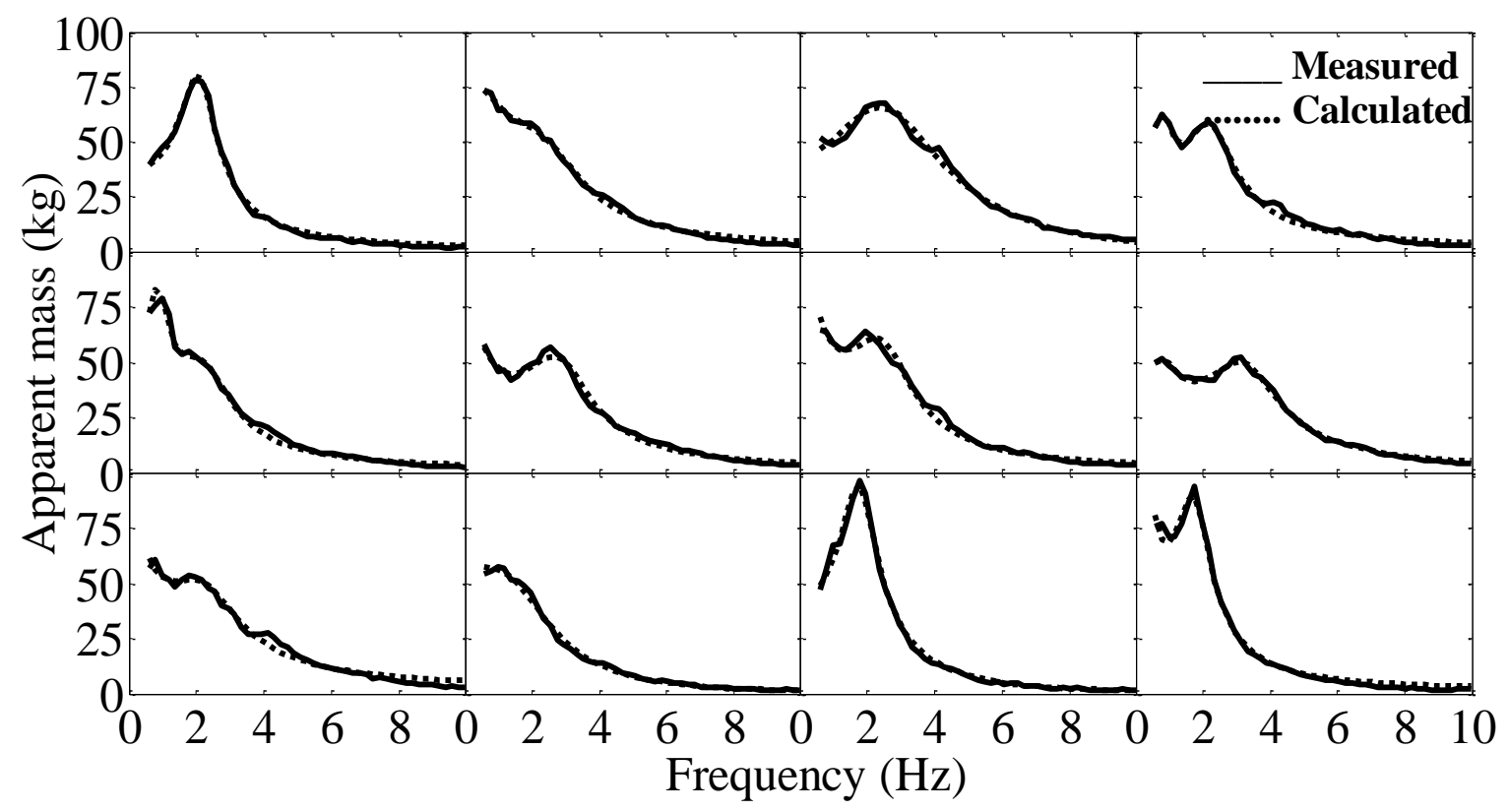

Figure 5. Apparent mass modulus of 12 subjects adopting the feet-supported position. 


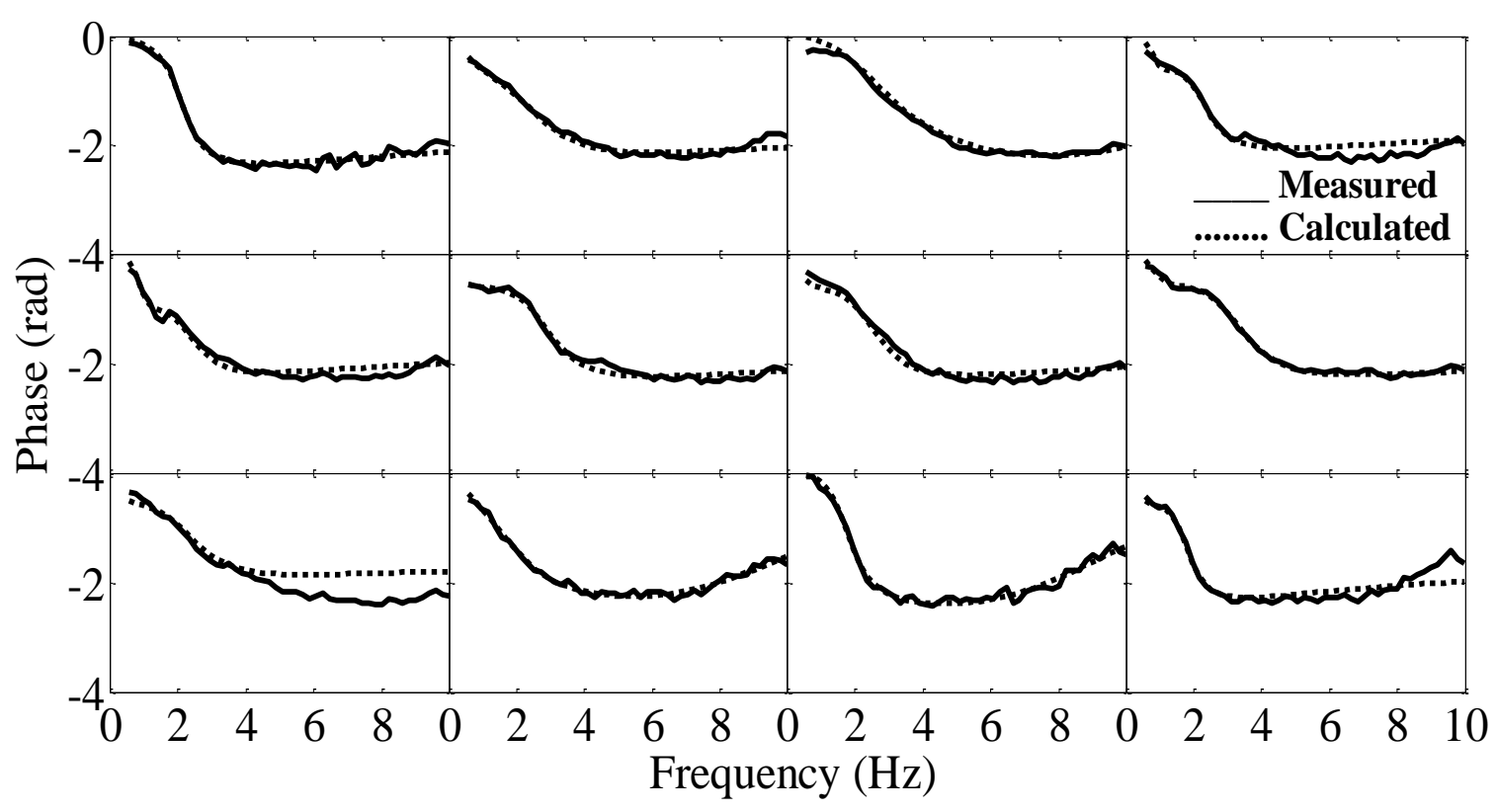

Figure 6. Apparent mass phase of 12 subjects adopting the feet-supported position.

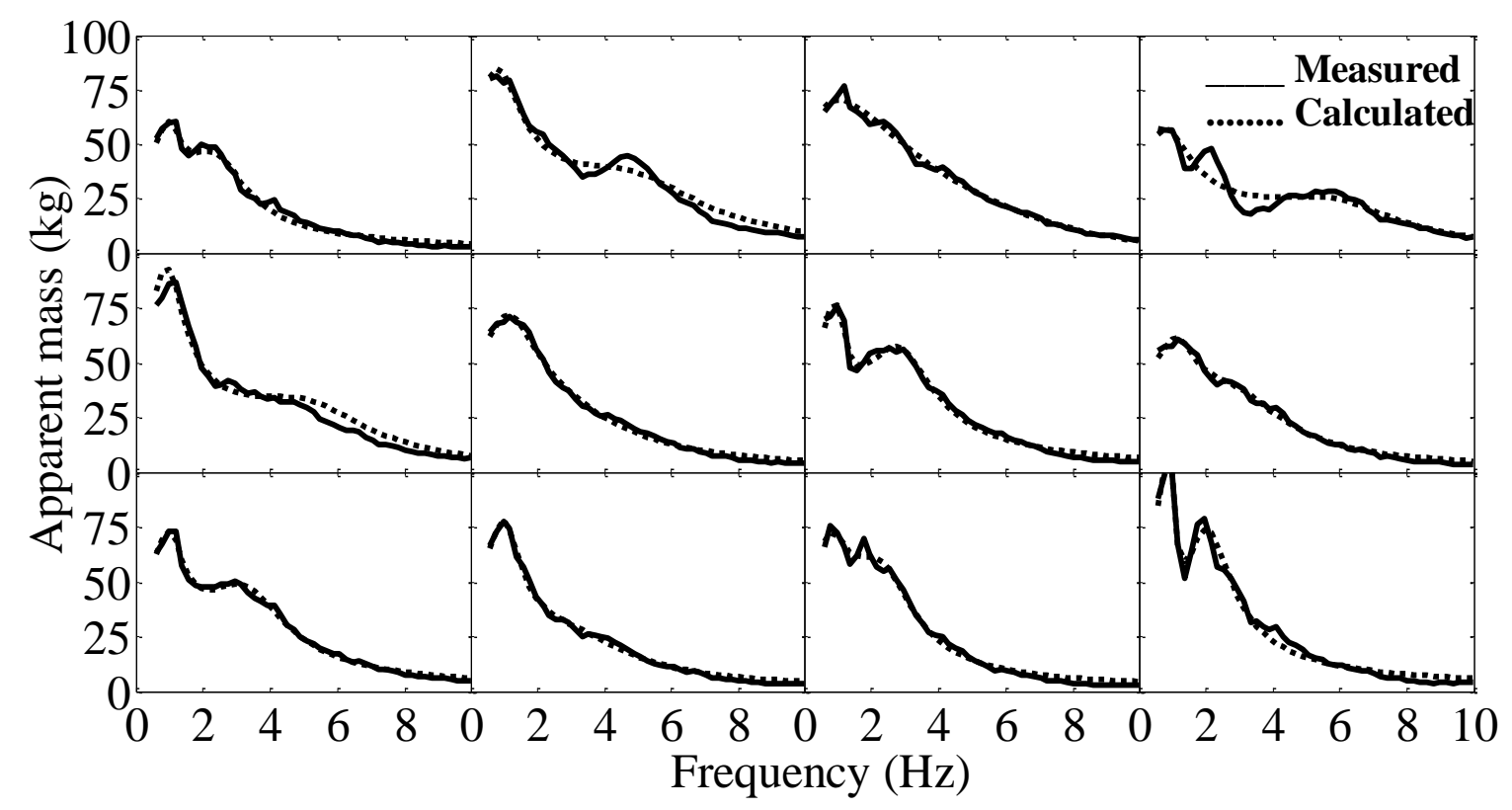

Figure 7. Apparent mass modulus of 12 subjects adopting the feet-unsupported position.

The parameters of the model obtained for each subject are shown in Table 1 for the feet-supported position and in Table 2 for the feet-unsupported position. The tables indicate high variability in the parameters among the subjects with both postures. This is expected given the high inter-subject variability shown in the experimental data, which may be attributed to the different masses and dimensions of the subjects used in the experiment. For both feet positions, more variability can be seen in the stiffness and damping coefficient of the rotational degrees-of-freedom (i.e. $k_{\mathrm{r}}$ and $c_{\mathrm{r}}$ ) than for the horizontal degrees-of-freedom (i.e. $k_{\mathrm{x}}$ and $c_{\mathrm{x}}$ ). While the order of magnitude of both $k_{\mathrm{x}}$ and $c_{\mathrm{x}}$ is similar among the subjects (except for subject 2 and subject 3 with the feet- 
supported position and subject 4 and subject 5 with the feet-unsupported position), the values of $c_{\mathrm{r}}$ ranged from units to hundreds and the values of $k_{\mathrm{r}}$ ranged from units to thousands. Previous models of the biodynamic responses to vertical whole-body vibration showed less variation in the optimised parameters than those reported in this study (e.g. $[22,23])$ owing to the lower inter-subject variability in the vertical apparent mass than in the fore-and-aft apparent mass. Unlike the fore-and-aft apparent mass, the vertical apparent mass curves are consistent between subjects with generally one single peak in the vicinity of $5 \mathrm{~Hz}$. Further discussion on the variation in the parameters of the model is presented in the sensitivity analysis section.

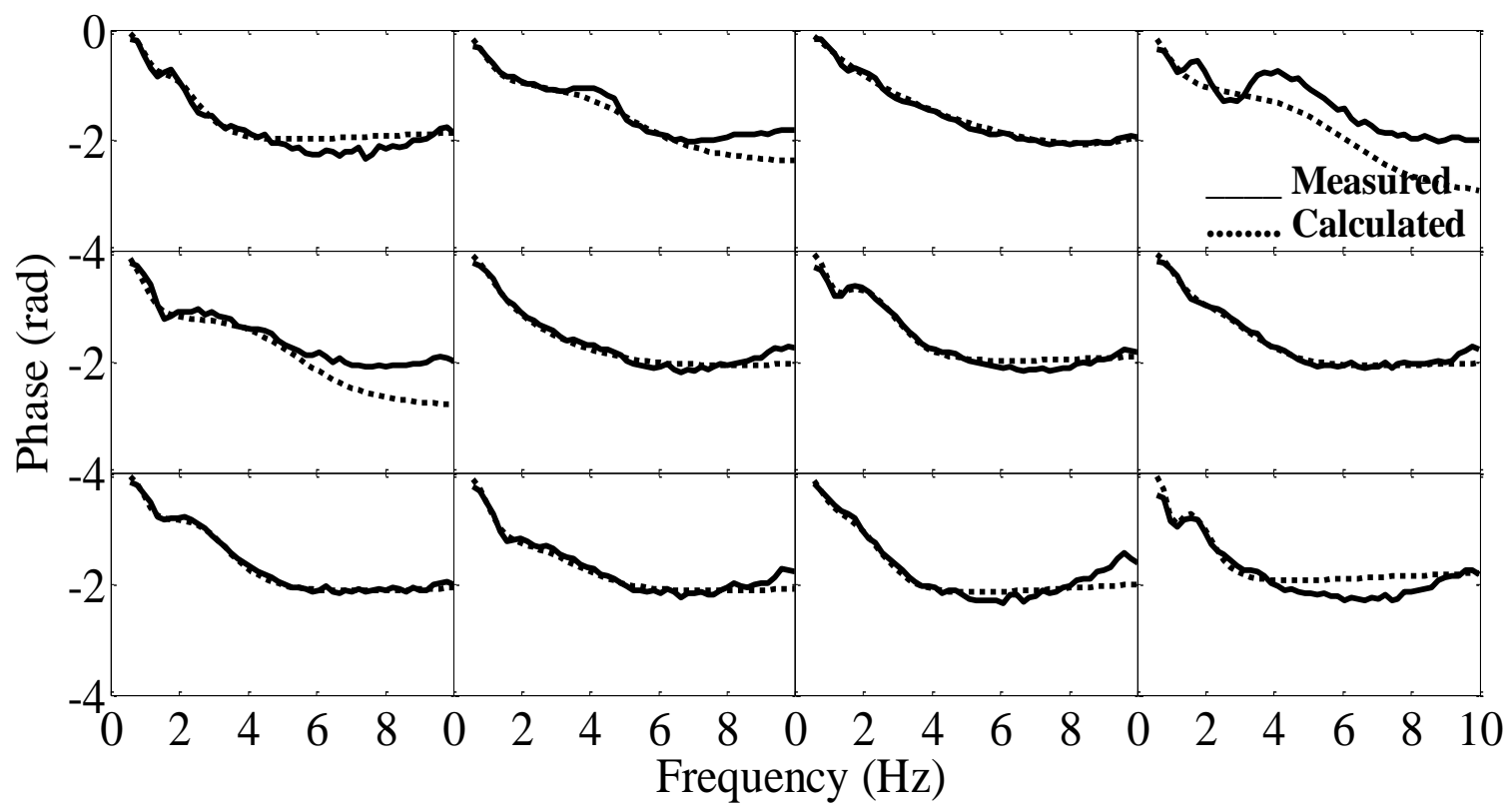

Figure 8. Apparent mass phase of 12 subjects adopting the feet-unsupported position.

\section{Sensitivity analysis}

The optimised parameters of the model were used to identify the contribution of each degree-of-freedom to the peaks of the fore-and-aft apparent mass. The parameters were allowed to vary one at a time by $\pm 40 \%$ of the optimised values. The studied parameters were $k_{\mathrm{x}}, c_{\mathrm{x}}, k_{\mathrm{r}}$ and $c_{\mathrm{r}}$. In general, the median or the mean responses are used for such an investigation. However, with a multi-peak response such as that shown here where the peaks happen at different frequencies for different subjects, the characteristics of the response might be concealed during the averaging process. Hence, sensitivity analysis was performed on individual data instead of the median data. The test was performed for different subjects to make sure that consistent results were obtained when using parameters from different subjects. Examples of the sensitivity analysis for two different subjects are shown in Figure 9 for the feet-supported position and in Figure 10 for the feet-unsupported position. Those subjects were chosen arbitrarily from the subjects who showed two peaks in their responses. Other subjects showed the same trend. The magnitude and frequency of the first peak were mainly affected by changes in the rotational degrees-of-freedom (i.e. $k_{\mathrm{r}}$ and $c_{\mathrm{r}}$ ), indicating that the first peak in the fore-andaft apparent mass is produced by a rotational vibration mode (Figures 9 and 10). This is consistent with previous studies, which reported that the first peak in the fore-and-aft apparent mass is produced by sway of the body when exposed to fore-and-aft vibration while sitting without a backrest $[10,24]$. This behaviour can be explained easily if one 
visualises the upper body on the seat as an inverted pendulum on a cart. When the cart (i.e. the seat) moves horizontally, the pendulum (i.e. the body) undergoes a sway motion. When a backrest was used, it provided a support for the body and the sway motion disappeared, as reported in $[11,24]$.
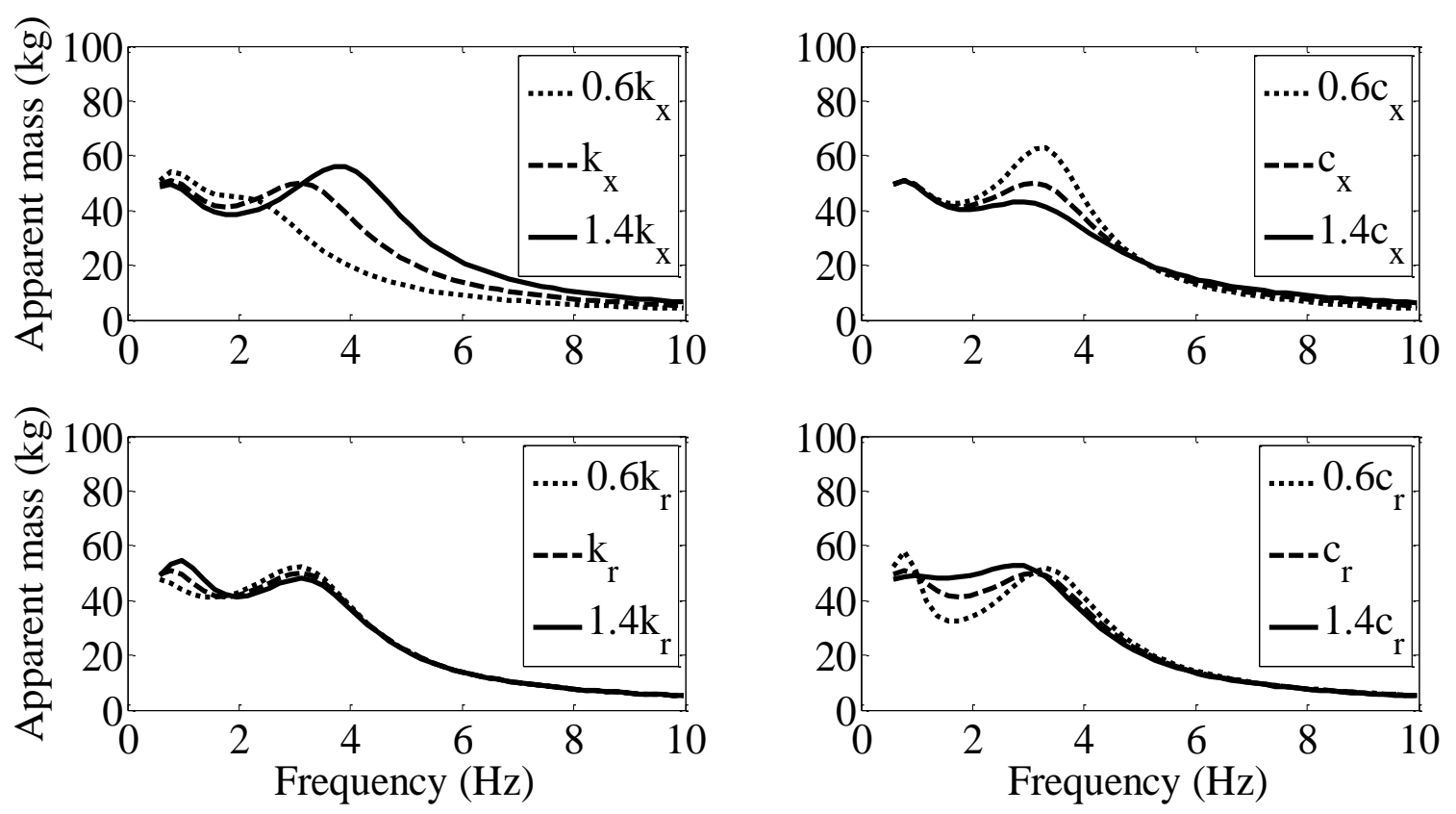

Figure 9. Changes in the apparent mass resulting from changes in the optimised parameters of the model. (Sensitivity analysis for the data of subject 8; feet-supported position.)
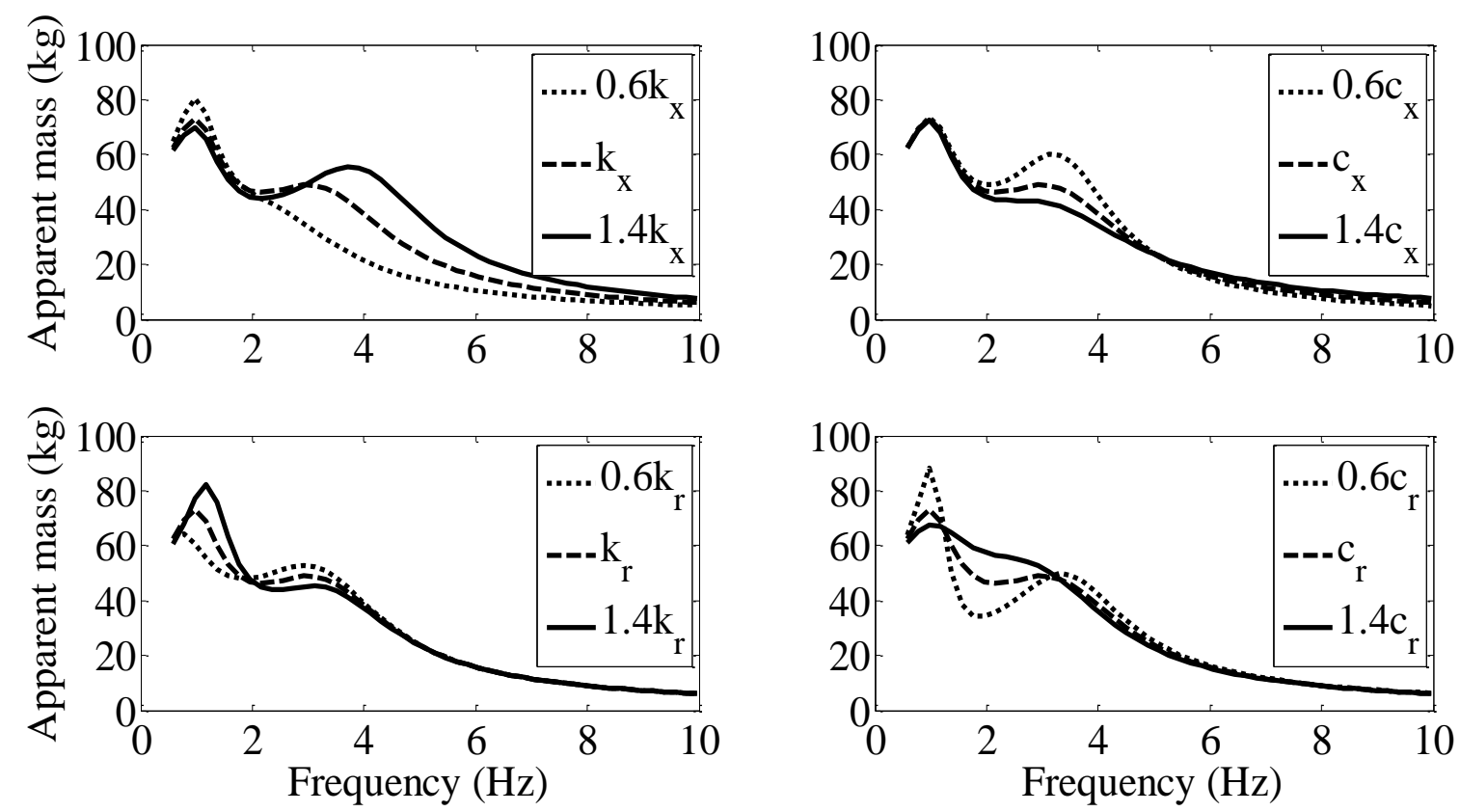

Figure 10. Changes in the apparent mass resulting from changes in the optimised parameters of the model. (Sensitivity analysis for the data of subject 9; feet-unsupported position.) 
The high variability in the parameters of the rotational degrees-of-freedom, $k_{\mathrm{r}}$ and $c_{\mathrm{r}}$ (Tables 1 and 2), could be attributed to the differences in the masses and dimensions of the subjects. The masses and dimensions of the subjects define the mass moment of inertia, the effect of which is embedded in the measured apparent mass, especially at low frequencies where rotational motion is dominant. This could be reflected in the optimised values of the rotational stiffness and damping owing to the effect of the mass moment of inertia on the rotational motion of mass 2 . The single rotational stiffness and single damping coefficient reported here can be thought of as the 'resultant' of the stiffness and damping coefficients of all the body parts that undergo rotational motion. The amount of rotation of those parts of the body is expected to depend on several factors, including, among others, body characteristics and sitting posture (e.g. erect posture, slouched posture). This dependency influences the apparent mass measured on the seat and consequently the optimised model parameters for each subject. Unlike the parameters reported in this work, parameters obtained from modelling the vertical apparent mass during vertical vibration did not show high variability between subjects (e.g. Nawayseh and Griffin [22]). This could be owing to the lower rotational motion of the body during vertical vibration than during fore-and-aft vibration.

Changes in the parameters of the translational degrees-of-freedom (i.e. $k_{\mathrm{x}}$ and $c_{\mathrm{x}}$ ) mainly affected the second peak of the fore-and-aft apparent mass with no or minimal effect on the first peak (Figure 9 and Figure 10). Hence, the second peak in the fore-andaft apparent mass is produced by a translational vibration mode. Although the proposed model is not a mechanistic model, this result may indicate that the second peak in the fore-and-aft apparent mass is produced by shear deformation of the tissue beneath the ischial tuberosities and/or beneath the thighs caused by translational motion of the body. In this case, the comfort of the driver and passengers could be affect by this motion. In addition, during long journeys and/or with high vibration magnitude, the excitation of the frequency of this peak could lead to health problems associated with the exposure to vibration [1]. Seats to be installed in vehicles that experience high fore-and-aft vibration should be designed to attenuate the vibration at the frequency of this peak.

The optimised value of $m_{1}$ was found to be either zero or very small for all individual and median data. This may imply that the mass directly on the seat (i.e. thighs or upper legs) undergoes rotational motion in addition to translational motion (which might have added to the variability discussed above): if the thighs (or upper legs) had no rotational motion, the model would have found some value for $m_{1}$ equivalent to the mass of the thighs (or upper legs) that will undergo only fore-and-aft motion. So, the model can be thought of as one single mass undergoing both translational and rotational motions representing both the resultant translational and resultant rotational motions of the different parts of the body. Previous studies have reported an effect of sitting posture and seating condition on the fore-and-aft apparent mass (e.g. [10,11,24]) and on the vibration transmitted through the seat to the driver and passengers [25]. Using a footrest or a backrest or changing the posture, for example from slouched to erect, may change the response owing to a possible change in body mass distribution, a change in the stiffness and/or a change in the damping, which could lead to different sets of model parameters with different postures or seating conditions. The non-linearity of the human body in response to vibration is another factor that can affect the apparent mass [14] and can lead to different sets of model parameters with different vibration magnitudes. Hence, the parameters of the model presented in this paper are applicable to the sitting postures and the vibration magnitude used in this study. More work should be carried out to identify the parameters of the model in other postures, such as that adopted by car drivers. 


\section{CONCLUSIONS}

In this work, a mathematical quantitative model that represents the fore-and-aft apparent mass of the human body has been developed. The model, which consisted of translational and rotational degrees-of-freedom, showed a close fit to the experimentally measured apparent mass. The model suggested that the whole mass on the seat, including the thighs/upper legs, undergoes both translational and rotational motion when exposed to fore-and-aft vibration. The model also suggested that the first peak of the fore-and-aft apparent mass is produced by a rotational vibration mode while the second peak of the fore-and-aft apparent mass is produced by a translational vibration mode. The proposed model can be integrated with a mathematical model of a vehicle seat to predict the transmissibility of the seat cushion. Alternatively, the model parameters can be used to build a mechanical dummy that can be used experimentally for seat testing applications instead of using a human subject, especially for testing under severe vibration conditions.

\section{ACKNOWLEDGEMENTS}

The author would like to thank the University of Sharjah for their financial and laboratory facilities.

\section{REFERENCES}

[1] Nuawi MZ, Ismail AR, Nor MJM, Rahman MM. Comparative Study of WholeBody Vibration Exposure Between Train and Car Passengers: A Case Study in Malaysia. International Journal of Automotive and Mechanical Engineering. 2011;4:490-503.

[2] Gong W, Griffin MJ. Measuring, evaluating and assessing the transmission of vibration through the seats of railway vehicles. Proceedings of the Institution of Mechanical Engineers, Part F: Journal of Rail and Rapid Transit. 2016: 09544097: 1-12.

[3] Griffin M. Handbook of Human Vibration (Academic, London). Chap. 1990;3:53-71.

[4] Lundström R, Lindberg L. Whole-body vibrations in road construction vehicles. 1983.

[5] Boileau P-É, Rakheja S. Whole-body vertical biodynamic response characteristics of the seated vehicle driver: measurement and model development. International Journal of Industrial Ergonomics. 1998;22:449-72.

[6] Holmlund P, Lundström R, Lindberg L. Mechanical impedance of the human body in vertical direction. Applied Ergonomics. 2000;31:415-22.

[7] Nawayseh N, Griffin M. Non-linear dual-axis biodynamic response to vertical whole-body vibration. Journal of Sound and Vibration. 2003;268:503-23.

[8] Mansfield N, Lundström R. Variability in the apparent mass of the seated person to horizontal vibration. United Kingdom Group Meeting on Human Response to Vibration; 1998.

[9] Paddan G, Griffin M. The transmission of translational seat vibration to the head-II. Horizontal seat vibration. Journal of biomechanics. 1988;21:199-206.

[10] Nawayseh N, Griffin M. Non-linear dual-axis biodynamic response to fore-andaft whole-body vibration. Journal of Sound and Vibration. 2005;282:831-62. 
[11] Nawayseh N, Griffin MJ. Tri-axial forces at the seat and backrest during wholebody fore-and-aft vibration. Journal of Sound and Vibration. 2005;281:921-42.

[12] Subashi G, Matsumoto Y, Griffin M. Modelling resonances of the standing body exposed to vertical whole-body vibration: Effects of posture. Journal of Sound and Vibration. 2008;317:400-18.

[13] Nawayseh N. Modelling of the biodynamic responses to whole-body vertical vibration. MESM'2008 (International Middle Eastern Multi-conference). Amman, Jordan: Philadelphia University; 2008.

[14] Mansfield N, Lundström R. Models of the apparent mass of the seated human body exposed to horizontal whole-body vibration. Aviation, space, and environmental medicine. 1999;70:1166-72.

[15] Stein GJ, Múčka P, Chmúrny R, Hinz B, Blüthner R. Measurement and modelling of $\mathrm{x}$-direction apparent mass of the seated human body-cushioned seat system. Journal of Biomechanics. 2007;40:1493-503.

[16] Qiu Y, Griffin MJ. Modelling the fore-and-aft apparent mass of the human body and the transmissibility of seat backrests. Vehicle system dynamics. 2011;49:70322.

[17] Karmarkar N. A new polynomial-time algorithm for linear programming. Proceedings of the sixteenth annual ACM symposium on Theory of computing: ACM. 1984;302-11.

[18] Potra FA, Wright SJ. Interior-point methods. Journal of Computational and Applied Mathematics. 2000;124:281-302.

[19] Griffin MJ. The validation of biodynamic models. Clinical Biomechanics. 2001;16:S81-S92.

[20] Ismail A, Abdullah S, Abdullah A, Deros BM. Whole-body vibration exposure of Malaysian taxi drivers. International Journal of Automotive and Mechanical Engineering. 2015;11:2786-92.

[21] Wei L. Predicting seat transmissibility from seat impedance and the apparent mass of the human body: University of Southampton; 2000.

[22] Nawayseh N, Griffin MJ. A model of the vertical apparent mass and the fore-andaft cross-axis apparent mass of the human body during vertical whole-body vibration. Journal of Sound and Vibration. 2009;319:719-30.

[23] Nawayseh N, Salalah O. Modelling of the biodynamic responses of the feet to vertical vibration. Middle Eastern Simulation and Modelling Conference (MESM'2011), Amman Open University (AOU), Amman, Jordan. 2011;14-6.

[24] Fairley T, Griffin M. The apparent mass of the seated human body in the foreand-aft and lateral directions. Journal of Sound and Vibration. 1990;139:299-306.

[25] Nawayseh N. Effect of the seating condition on the transmission of vibration through the seat pan and backrest. International Journal of Industrial Ergonomics. 2015;45:82-90. 